\title{
CALIDAD DEL PALMITO EN FUNCIÓN DE LA FERTILIZACIÓN Y DEL ACONDICIONAMIENTO POSCOSECHA ${ }^{1}$
}

\author{
Francisco Paulo Chaimsohn ${ }^{2}$, Ruth de La Asunción-Romero ${ }^{3}$, Jorge Mora-Urpí ${ }^{4}$,Enrique Villalobos-Rodríguez ${ }^{5}$
}

\section{RESUMEN}

Calidad del palmito en función de la fertilización y del acondicionamiento poscosecha. Con el objetivo determinar el efecto del tipo de fertilización, del tipo de empaque y de la temperatura sobre la calidad del palmito al natural, en enero de 2005, en Guápiles, Costa Rica, se evaluó la respuesta del palmito al efecto de la fertilización química y orgánica, dos tipos de empaque (bolsa de polietileno de baja densidad termosoldada y bandeja de poliestireno expandido cubierta con película de policloruro de vinilo) y de tres temperaturas (cinco, 10 y $20{ }^{\circ} \mathrm{C}$ ). El palmito fertilizado orgánicamente presentó una mejor apariencia general y acidez que el producido bajo la fertilización química (pH 6,45 vs. 6,24, respectivamente) durante los primeros seis días en almacenamiento, pero sufrió una reducción en la apariencia general, un aumento en la coloración amarillenta y en el pH a los 15 días; además, produjo olores desagradables a partir de los 12 días. El palmito empacado en las bolsas plásticas a $5{ }^{\circ} \mathrm{C}$ se conservó en buenas condiciones hasta el final del almacenamiento. La bandeja de poliestireno cubierta con plástico fue inapropiada para la conservación del producto.

Palabras clave: Bactris gasipaes, fertilización orgánica, empaque, temperatura, almacenamiento.

\begin{abstract}
Peach palm heart quality in relation to chemical fertilization and post-harvest conditioning. The objective of this work was to evaluate the effect of fertilization, packing type, and temperature on palm heart quality. The work, carried out in January, 2005, evaluated the post harvest quality of peach palm heart in response to two fertilization procedures: chemical and organic fertilizer; two packing procedures: a thermosealed low-density polyethylene bag and a tray of expanded polystyrene, covered with a film of polyvinyl chloride): and three temperatures: 5,10 and $20^{\circ} \mathrm{C}$. The palm heart fertilized organically showed better appearance and acidity than the inorganic product ( $\mathrm{pH} 6,45$ vs 6,24, respectively) during the first six days in storage (dis). However, it showed a decline in appearance, including yellowing, acidification, and undesirable odors after 12 dis. The palm heart packed in plastic bags at $5{ }^{\circ} \mathrm{C}$ was properly preserved until the end of the storage period. The tray of expanded polystyrene was not appropriate for the preservation of peach palm.
\end{abstract}

Key words: Bactris gasipaes, organic fertilization, packing methods, temperature, storage.

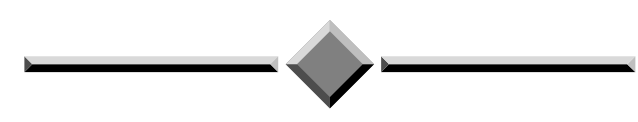

\section{INTRODUCCIÓN}

Durante los últimos años se ha presentado una tendencia global de cambio en los hábitos del consumidor hacia una forma de vida más saludable, con un incremento en la proporción de frutas y vegetales frescos en la dieta. Esto se refleja en una mayor adquisición de productos frescos en su estado natural o con proceso mínimo (De la Asunción et al. 1995, Garett 2002). El palmito de pejibaye (Bactris

\footnotetext{
1 Recibido: 5 de enero, 2009. Aceptado: 16 de noviembre, 2009. Parte de la tesis de doctorado en Sistemas de Producción Agrícola Tropical Sostenible, Facultad de Ciencias Agroalimentarias, Universidad de Costa Rica.

2 Instituto Agronômico do Paraná (IAPAR). Becado de Doctorado en el exterior por el CNPq - Brasil. fpchaimsohn@yahoo.com.br

3 Centro de Investigación en Tecnología de Alimentos, Universidad de Costa Rica. San José, Costa Rica. ruth.delaasuncion@ucr.ac.cr

4 In memorian. Escuela de Biología, Universidad de Costa Rica. San José, Costa Rica.

5 Centro de Investigación en Granos y Semillas, CIGRAS, Universidad de Costa Rica. San José, Costa Rica. villalobos.enrique@gmail.com
} 
gasipaes Kunth) no escapa de esta tendencia mundial y actualmente se considera que existe una demanda insatisfecha de este producto al natural (Córdoba 1995, Clement et al. 1996, Velázquez et al. 2001, Anefalos et al. 2007). Cuando está fresco, el palmito de pejibaye presenta entre 0,89 y $1,05 \%$ de fibra, de 2,3 a $2,4 \%$ de proteína cruda, 2,3\% de azúcares totales y 1,4\% de azúcares reductores, $0,59 \mathrm{~g} \mathrm{100/g}$ de ácido cítrico, $3,2 \%$ de vitamina C y 0,88 g 100/g de ácido acético (Ferreira 1982, De la Asunción 1992).

Además de atender una demanda existente y potencial, la producción de palmito de pejibaye para su comercialización como producto al natural, o con proceso mínimo, es una actividad con gran potencial, principalmente para pequeños agricultores (Chaimsohn 2000, Mora Urpí 2002). La comercialización de este tipo de producto promueve la apertura de mercados alternativos en el ámbito local, nacional e internacional, adicionalmente al mercado existente para el producto en conserva, disminuyendo o minimizando la dependencia del agricultor con la industria (Chaimsohn 2000).

El palmito de pejibaye presenta una ventaja, con relación los palmitos de palmas del género Euterpe, para la comercialización como producto fresco o mínimamente procesado, porque posee bajos contenidos de las enzimas que causan la oxidación del palmito, además de tener menor contenido de taninos y más azúcares (Ferreira et al. 1982, Clement y Bovi 1999).

El mercado actual de palmito al natural es relativamente incipiente aún, pero con gran potencial de crecimiento. Asimismo, su producción orgánica agregaría un mayor valor, principalmente en los mercados donde hay una demanda creciente de productos orgánicos. La tendencia mundial al consumo de éstos también es ascendente (Kortbech-Olesen 2002).

El palmito para consumo al natural requiere tecnología de producción diferenciada en comparación con el destinado a la industria, dado que su procesamiento y comercialización son distintos, principalmente para mercados que exigen una alta calidad. La producción de palmito orgánico podría agregar valor al producto y facilitar su comercialización en nichos de mercado con preferencia por este tipo de productos (Chaimsohn et al. 2007). Luego, es importante evaluar los efectos de la fertilización orgánica sobre la calidad del palmito al natural.

Además, para el establecimiento y desarrollo de mercados es de fundamental importancia definir y garantizar parámetros de calidad, además de desarrollar tecnologías y procesos de manejo en el campo y en poscosecha que garanticen un producto de calidad, y con un máximo de vida de estante.

Los principales factores de calidad en los alimentos en general, y en los vegetales "mínimamente procesados", son la apariencia, el sabor, la textura (firmeza, crujencia y dureza), y la calidad nutricional (Bourne 2002, Kader 2002). Asimismo, el "procesamiento mínimo" debe ser hecho buscando obtener un producto que mantenga las características del vegetal fresco y sea atractivo y seguro para el consumidor. El producto debe ser conservado bajo condiciones de refrigeración y empacado para mantener su calidad y evitar la contaminación con agentes biológicos y químicos.

Específicamente en relación con el palmito al natural, Calvo (2000) señala, como principales problemas de calidad, la textura (palmito duro), además del sabor y la apariencia, que son afectados por la exposición al sol del palmito cosechado. Otras características de calidad son la apariencia general, el color, la ausencia de daño microbiano, y la ausencia de olores extraños. Hay muy pocos trabajos en la literatura sobre la calidad del palmito para consumo al natural.

Este trabajo tuvo como objetivo determinar el efecto del tipo de fertilización, de empaque y de la temperatura sobre la calidad del palmito al natural.

\section{MATERIALES Y MÉTODOS}

La siembra experimental fue establecida el 2 y 3 de octubre del 2003 en la Estación Experimental Los Diamantes de la Universidad de Costa Rica, ubicada en Guápiles, cantón Pococí, provincia de Limón; Costa Rica, coordenadas $10^{\circ} 22^{\prime}$ latitud norte y $83^{\circ} 76^{\prime}$ ' longitud oeste. Se usó la variedad de pejibaye sin espinas 'Diamantes 10', a una densidad de 6.666 plantas/ha. El suelo de la parcela experimental es un Andic Oxyaquic Dystrudepts, de textura franco arenosa. La temperatura promedio mensual fue de $23,9{ }^{\circ} \mathrm{C}$ y la precipitación anual fue de $4.719 \mathrm{~mm}$, con picos en mayo y noviembre/diciembre. Detalles de las labores culturales, diseño experimental y otros del ensayo de campo se dan en el artículo de Chaimsohn et al. (2007).

A partir del octavo mes se aplicó, trimestralmente, abonos químicos y orgánicos, de acuerdo con los tratamientos de fertilización (Cuadro 1).

AGRONOMÍA MESOAMERICANA 20(2):195-209. 2009 
Cuadro 1. Fertilizantes químicos y enmiendas orgánicas aplicados experimentalmente al palmito, en diferentes meses después de la siembra (mds). Costa Rica. 2005.

\begin{tabular}{|c|c|c|c|}
\hline \multirow[t]{2}{*}{ mds* } & \multirow[t]{2}{*}{ Material** } & \multicolumn{2}{|c|}{ Fertilización (g/planta) } \\
\hline & & química & orgánica \\
\hline \multirow{2}{*}{2} & fosfato diamónico - DAP & 40,0 & - \\
\hline & boñiga & - & 2.000 \\
\hline \multirow{2}{*}{8} & $18-5-15-6-0,7$ & 30,0 & - \\
\hline & lombri compost $(+)$ & - & 1.200 \\
\hline \multirow{3}{*}{11} & nitrato de amonio & 30,0 & - \\
\hline & lombri compost $(++)$ & - & 2.000 \\
\hline & $18-5-15-6-0,7$ & 100,0 & - \\
\hline 14 & $\begin{array}{l}\text { compost de palma de } \\
\text { aceite }(+++)\end{array}$ & - & 2.500 \\
\hline
\end{tabular}

* meses después de la siembra.

** (+) compost de la Estación Experimental de Ochomogo - Universidad de Costa Rica (UCR); (++) compost de Hacienda Ganadera Pozo Azul, La Virgen, Sarapiquí; (+++) compost de desecho de palma de aceite.

\section{Corte, transporte y almacenamiento}

El palmito del tallo primario se cosechó cuando las palmas tenían 473 días de sembradas en el campo. El estípete tenía un diámetro promedio de aproximadamente $12 \mathrm{~cm}$ y una altura promedio de 163 y 172 $\mathrm{cm}$, de las cepas fertilizadas con enmienda orgánica y abonos químicos, respectivamente. La cosecha se realizó entre las 6 y las 9 am, bajo condiciones de alta humedad y a una temperatura entre 20 a $22{ }^{\circ} \mathrm{C}$.

Después del corte, los palmitos fueron cuidadosamente acomodados sobre hojas cortadas para evitar el contacto con el suelo. Los tallos fueron transportados a temperatura ambiente (alrededor de $20{ }^{\circ} \mathrm{C}$ ) durante aproximadamente 90 minutos. Los palmitos fueron almacenados en una cámara fría de la planta del Centro Nacional de Ciencia y Tecnología de Alimentos (CITA) de la Universidad de Costa Rica, a una temperatura de $10{ }^{\circ} \mathrm{C}$, para ser procesados al día siguiente.

\section{Procesamiento y montaje del experimento}

Los palmitos de las parcelas con fertilización química y orgánica fueron procesados separadamente, siguiendo el diagrama de flujo (Figura 1).

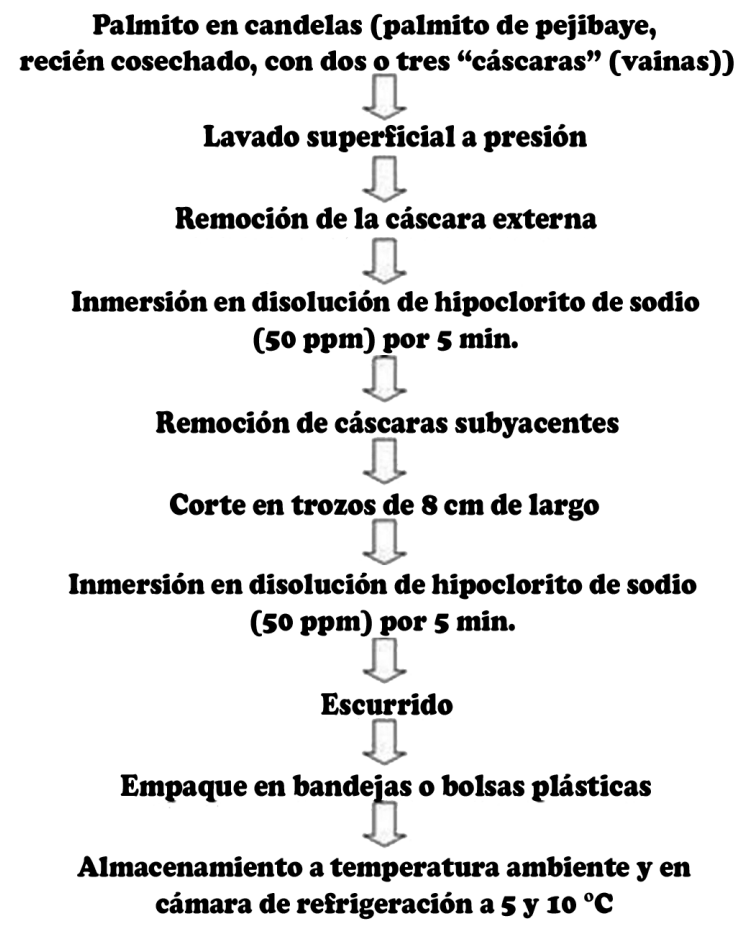

Figura 1. Diagrama de flujo del procesamiento del palmito en la planta agroindustrial del Centro Nacional de Ciencia y Tecnología de Alimentos (CITA). Costa Rica. 2005.

Nota: Para el lavado superficial a presión se utilizó una manguera corriente, conectada a un tubo de cocina normal.

En cada fase se usó una mesa con superficie de acero inoxidable. Las mesas, cuchillos y otros utensilios utilizados en el procesamiento fueron higienizados con disolución de hipoclorito de sodio a 50 ppm, preparada con una solución sanitizante al $12 \%$.

Los palmitos fueron lavados superficialmente con agua potable a presión. En seguida se removió la cáscara (vaina) externa, y fueron sumergidos en disolución de hipoclorito de sodio a $50 \mathrm{ppm}$, preparada con una solución sanitizante al $12 \%$. Luego se eliminaron las vainas subyacentes y los palmitos foliares fueron cortados en trozos de $8 \mathrm{~cm}$ de largo. Los pedazos fueron sumergidos en una disolución de hipoclorito de sodio a $50 \mathrm{ppm}$, por cinco minutos. Antes del empaque, los trozos fueron colocados en una canasta metálica para retirar el exceso de líquido.

Se utilizaron dos tipos de empaque: bolsas plásticas de polietileno de baja densidad $(26 \times 17,5 \mathrm{~cm})$, 
las cuales fueron termosoldadas posteriormente, y bandejas de poliestireno expandido ("esterofón") (13,5 x 13,5 cm), cubiertas con película plástica transparente (película de policloruro de vinilo PVC, con un espesor de $11 \pm 0,5 \mu \mathrm{m})$. Se colocaron tres trozos de palmito en cada empaque. Cada empaque con el producto fue pesado en una balanza electrónica y luego colocados en una cámara refrigerada a 5 y $10{ }^{\circ} \mathrm{C}(20$ empaques por temperatura y tratamiento de fertilización). También se dejaron 10 empaques por tratamiento a temperatura ambiente $\left(20 \pm 2{ }^{\circ} \mathrm{C}\right)$, ya que es muy común la comercialización del palmito al natural en ferias agrícolas y otros mercados nacionales.

\section{Variables evaluadas}

Se evaluaron las siguientes variables: pérdida de peso de la muestra, apariencia general, daño microbiano, presencia de olores extraños, textura, color y $\mathrm{pH}$. Las muestras almacenadas en bolsas y en bandejas, a 5 y $10{ }^{\circ} \mathrm{C}$, fueron evaluadas a los seis, nueve, 12 y 15 días en almacenamiento (dea). A excepción de la pérdida de peso, las demás variables también fueron determinadas al día siguiente de la cosecha.

\section{Pérdida de peso de las muestras}

Para cada tratamiento (empaque $\mathrm{x}$ temperatura) se tomaron cinco muestras al azar y su peso fue determinado en una balanza digital, para registrar una eventual pérdida del mismo.

Inmediatamente después de la determinación del peso, las muestras fueron evaluadas en relación con la apariencia general, el daño microbiano y la presencia de olores raros, usando una escala de uno a cinco, como se describe a continuación.

\section{Apariencia general}

- Nota 5: producto muy similar al palmito recién cosechado.

- Nota 4: ligeramente inferior al producto fresco; presenta tonalidad del color levemente más oscuro, sin alteraciones importantes en la textura superficial.

- Nota 3: inferior al producto fresco; presenta cambios en la tonalidad (color más oscuro), manchas amarillentas en aproximadamente $50 \%$ de la superficie del producto, rugosidades y/o sustancias mucilaginosas; producto con restricciones importantes para el consumo.

- Nota 2: muy inferior al producto fresco; presenta cambios acentuados en la tonalidad del color, manchas en más de $50 \%$ de la superficie del producto, rugosidades y/o sustancias mucilaginosas; producto sin condiciones para el consumo.

- Nota 1: caracterizado como un producto totalmente deteriorado en su color y textura superficial.

\section{Daño microbiano}

- Nota 5: producto muy similar al palmito recién cosechado.

- Nota 4: producto con un máximo de tres colonias muy pequeñas de hongos y/o bacterias (HB) en la superficie.

- Nota 3: producto medianamente infectado (hasta un tercio de la superficie) con colonias de HB.

- Nota 2: producto muy infectado con colonias de HB (entre un tercio y la mitad de la superficie del producto).

- Nota 1: producto con más de la mitad de la superficie invadida por colonias de HB.

\section{Olores extraños}

- Nota 5: producto muy similar al palmito recién cosechado; olor característico de vegetal fresco recién cortado, levemente dulcificado.

- Nota 4: producto con una presencia leve de olores ligeramente agrios y/o alcohólicos, pero aún factible de ser comercializado.

- Nota 3: producto con olores agrios y/o alcohólicos acentuados y con serias restricciones para la comercialización y consumo.

- Nota 2: producto con olores muy desagradables, agrios y/o alcohólicos muy acentuados; presencia de olores característicos de la degradación de proteínas y aminoácidos e inicio de la descomposición microbiana y por lo tanto inapropiado para el consumo.

- Nota 1: producto con olores característicos de la avanzada descomposición microbiana.

\section{Textura}

Las muestras fueron cortadas en trozos de aproximadamente $3 \mathrm{~cm}$ de largo y la textura fue determinada 
por la resistencia al corte, con el equipo "Texture Analyser TAXT Plus". Se usaron cinco trozos por muestra. Se empleó un programa del equipo, previamente validado para la evaluación de la textura del palmito, con las siguientes especificaciones: método: compresión; velocidad: $10 \mathrm{~mm} / \mathrm{seg}$; acción sobre el objeto: tensión; distancia: $10 \mathrm{~mm}$; tensión: 100\%; tipo del disparador: auto (fuerza); fuerza del disparador: 0,09807 N; distancia del disparador: $2 \mathrm{~mm}$; sensibilidad del corte: $0,09807 \mathrm{~N}$.

Color

Se cortaron pequeñas láminas de la parte externa de los trozos de palmito, y el color fue determinado con un equipo Color Flex, que utiliza el sistema cromático Hunter. Este sistema se representa en coordenadas rectangulares, con los valores de parámetro "a" en las abscisas, los valores "b" en ordenadas y la luminosidad "L" en un tercer eje perpendicular. Los tres ejes representan las variaciones de las funciones rojoverde $(+a,-a)$, amarillo-azul (+b,-b) y blanco-negro o claro-oscuro (L) que corresponden a las sensaciones que el cerebro humano recibe de los nervios ópticos (Hunterlab 1996). Estos valores se ordenan tridimensionalmente en la representación que se conoce como "Sólido de Hunter". Se efectuó una lectura por muestra porque, en pruebas anteriores, se constató que el coeficiente de variación, al evaluar el color del palmito de pejibaye, resultó muy bajo.

Se calculó la "diferencia total de color" $(\Delta \mathrm{E})$ mediante la ecuación que se presenta abajo (Hunterlab 1996). La $\Delta \mathrm{E}$ indica cuánto se aleja el color de una determinada muestra del patrón (Hunterlab 1996, Good s.f.); o sea, cuanto mayor el valor del $\Delta \mathrm{E}$, mayor es la diferencia del color de la muestra con relación a un patrón; en este caso, el patrón se refiere al palmito recién cosechado.

$$
\Delta E=\sqrt{(\Delta L)^{2}+(\Delta a)^{2}+(\Delta b)^{2}}
$$

donde:

$\Delta \mathbf{L}=$ (valor $\mathbf{L}$ la muestra) - (valor $\mathbf{L}$ "estándar")

$\Delta \mathbf{a}=$ (valor a la muestra) - (valor a "estándar")

$\Delta \mathbf{b}=$ (valor b la muestra) - (valor b "estándar")

Nota: se consideró como valores estándar, los valores promedio, de cada parámetro, observados en el primer día, separadamente para el palmito con fertilización química y orgánica.

Acidez

Se tomaron muestras de $500 \mathrm{~g}$ de palmito recién cosechado y cultivado con fertilizante orgánico y químico. Las muestras se mantuvieron en refrigeración hasta el día siguiente, cuando se determinó el pH. El $\mathrm{pH}$ también fue determinado a los seis y 15 días de al macenamiento, en una pasta de palmito con agua en una proporción 1:1.

\section{Análisis estadístico de los datos}

Se usó un arreglo factorial (dos tipos de fertilización $\mathrm{x}$ dos tipos de empaque $\mathrm{x}$ tres temperaturas) en un diseño experimental irrestricto al azar; los datos fueron analizados utilizando el programa de análisis estadístico SAS ${ }^{\circledR}$.

\section{RESULTADOS}

\section{Pérdida de peso}

La variación en el peso inicial del palmito (recién cosechado) entre las muestras fue de 10 a $12 \%$. Mediante un análisis de covarianza se determinó que esta variable no influyó en la pérdida de peso del palmito. Tampoco se comprobó algún efecto de la fertilización sobre esta variable, como se ha observado en algunas hortalizas (Santos et al. 2001).

El palmito empacado en las bandejas, presentó una pérdida de peso promedio más acelerada en el tiempo y hasta diecisiete veces mayor que aquella observada en el palmito empacado en bolsas de polietileno (Cuadro 2). El empacado en bandejas presentó pérdida de peso creciente en función de la temperatura, en el período de almacenamiento en el cual fue evaluado. Mientras que, el producto empacado en bolsas y almacenado a 5 y $10{ }^{\circ} \mathrm{C}$ no presentó variaciones importantes en su peso (interacción empaque $\mathrm{x}$ temperatura, $\mathrm{P}$ $<0,001)$. Incluso, el producto empacado en bandejas y mantenido a $5{ }^{\circ} \mathrm{C}$ presentó una tendencia a una mayor pérdida de peso que el palmito almacenado en bolsas a $10{ }^{\circ} \mathrm{C}$, lo que pone en evidencia la importancia del empaque en el mantenimiento de la calidad del palmito para consumo al natural. 
Cuadro 2. Pérdida de peso (\%) del palmito al natural, en función del tipo de fertilización, del empaque y de la temperatura, a los seis, nueve, 12 y 15 días de almacenamiento. Costa Rica. 2005.

\begin{tabular}{|c|c|c|c|c|c|c|}
\hline \multirow{2}{*}{$\begin{array}{c}\text { Fertilización } \\
\text { (F) }\end{array}$} & \multirow{2}{*}{$\begin{array}{l}\text { Empaque } \\
\text { (E) }\end{array}$} & \multirow{2}{*}{$\begin{array}{c}\text { Temperatura } \\
{ }^{\circ} \mathbf{C}(\mathbf{T})\end{array}$} & \multicolumn{4}{|c|}{ Días en almacenamiento } \\
\hline & & & 6 & 9 & 12 & 15 \\
\hline \multirow{6}{*}{ Química } & \multirow{3}{*}{ Bandeja } & 5 & 0,9 & 0,8 & 1,3 & 1,6 \\
\hline & & 10 & 0,8 & 1,4 & 1,2 & 1,8 \\
\hline & & 20 & 2,9 & 3,6 & - & - \\
\hline & \multirow{3}{*}{ Bolsa } & 5 & 0,4 & 0,4 & 0,5 & 0,6 \\
\hline & & 10 & 0,0 & 0,2 & 0,4 & 0,3 \\
\hline & & 20 & 0,7 & 0,6 & - & - \\
\hline \multirow{6}{*}{ Orgánica } & \multirow{3}{*}{ Bandeja } & 5 & 0,7 & 1,0 & 1,4 & 1,7 \\
\hline & & 10 & 0,8 & 1,0 & 1,3 & 1,7 \\
\hline & & 20 & 2,7 & 4,1 & - & - \\
\hline & \multirow{3}{*}{ Bolsa } & 5 & 0,1 & 0,3 & 0,5 & 0,2 \\
\hline & & 10 & 0,2 & 0,1 & 0,3 & 0,1 \\
\hline & & 20 & 1,9 & 0,7 & - & - \\
\hline \multicolumn{7}{|l|}{ ANDEVA } \\
\hline $\mathrm{F}$ & & & $\mathrm{ns}$ & $\mathrm{ns}$ & ns & $\mathrm{ns}$ \\
\hline $\mathrm{E}$ & & & $* *$ & $* *$ & $* *$ & $* *$ \\
\hline $\mathrm{T}$ & & & $* *$ & $* *$ & $\mathrm{~ns}$ & ns \\
\hline $\mathrm{E}^{*} \mathrm{~T}$ & & & $*$ & $* *$ & ns & $\mathrm{ns}$ \\
\hline
\end{tabular}

Simbología: $\mathrm{ns}=(\mathrm{P}>0,05) ; *=(\mathrm{P}<0,05) ; * *=(\mathrm{P}<0,001) ;-=$ Lectura omitida por deterioro del producto.

\section{Análisis sensorial}

A los seis días de almacenamiento, el palmito proveniente de plantas fertilizadas con enmienda orgánica presentó una calificación referente a la apariencia general ligeramente superior; mientras que, a los 15 días el palmito cosechado de plantas fertilizadas químicamente presentó una calificación levemente mayor $(\mathrm{P}<0,017)$ (Cuadro 3$)$.

El almacenamiento en bolsas fue superior que el empaque en bandejas para el mantenimiento de la apariencia general aceptable del palmito $(\mathrm{P}<0,001)$. La apariencia general disminuyó con el incremento en la temperatura $(\mathrm{P}<0,001)$. Además, se observó un efecto de la interacción empaque $\mathrm{x}$ temperatura $(\mathrm{P}<$ $0,001)$ en la apariencia general (Cuadro 3). A los nueve días el producto empacado en bolsa plástica presentó mejor apariencia general que el palmito almacenado en las bandejas, a 5 y $10{ }^{\circ} \mathrm{C}$; mientras que, a $20{ }^{\circ} \mathrm{C}$ el producto empacado en bandeja y bolsa presentó importante pérdida en la apariencia general. A los 15 dea, el producto empacado en bolsa y almacenado a 5 y 10 ${ }^{\circ} \mathrm{C}$ también presentó mejor apariencia general, con relación al palmito empacado en bandejas (Cuadro 3).

El tipo de fertilización, en el cultivo del palmito, no influyó en el daño microbiano del producto, como sí ocurrió con el tipo de empaque y la temperatura. La temperatura más alta y las bandejas así como la combinación de ambos factores (interacción empaque $\mathrm{x}$ temperatura, $\mathrm{P}<0,001$ ) resultaron detrimentales para la prevención del daño microbiano en el palmito. Hasta el noveno día, solamente el producto almacenado 
Cuadro 3. Apariencia general, daño microbiano y presencia de olores extraños del palmito al natural, en función del tipo fertilización, del empaque y de la temperatura, a los 6, 9, 12 y 15 días de almacenamiento. Costa Rica. 2005.

\begin{tabular}{|c|c|c|c|c|c|c|c|c|c|c|c|c|c|c|}
\hline \multirow[t]{2}{*}{$\begin{array}{l}\text { Fertiliza- } \\
\text { ción }(\mathbf{F})\end{array}$} & \multirow[t]{2}{*}{$\begin{array}{l}\text { Empaque } \\
\text { (E) }\end{array}$} & \multirow[t]{2}{*}{$\mathrm{T}^{\circ} \mathrm{C}$} & \multicolumn{4}{|c|}{ Apariencia general } & \multicolumn{4}{|c|}{$\begin{array}{c}\text { Daño microbiano } \\
\text { (Días en almacenamiento) }\end{array}$} & \multicolumn{4}{|c|}{ Olores extraños } \\
\hline & & & 6 & 9 & 12 & 15 & 6 & 9 & 12 & 15 & 6 & 9 & 12 & 15 \\
\hline \multirow{6}{*}{ Química } & \multirow{3}{*}{ Bandeja } & 5 & 4,4 & 4,3 & 4,2 & 3,7 & 5,0 & 5,0 & 5,0 & 4,4 & 5,0 & 4,5 & 4,5 & 3,9 \\
\hline & & 10 & 4,4 & 4,3 & 3,5 & 1,5 & 5,0 & 5,0 & 3,9 & 2,6 & 5,0 & 4,5 & 4,2 & 3,4 \\
\hline & & 20 & 1,5 & 1,3 & - & - & 5,0 & 4,2 & - & - & 5,0 & 2,8 & - & - \\
\hline & \multirow{3}{*}{ Bolsa } & 5 & 4,2 & 4,5 & 4,3 & 4,4 & 5,0 & 4,8 & 5,0 & 5,0 & 5,0 & 4,7 & 4,5 & 4,5 \\
\hline & & 10 & 4,4 & 4,5 & 4,2 & 4,0 & 5,0 & 5,0 & 5,0 & 5,0 & 5,0 & 5,0 & 4,6 & 4,5 \\
\hline & & 20 & 3,0 & 3,1 & - & - & 5,0 & 4,8 & - & - & 4,4 & 3,5 & - & - \\
\hline \multirow{6}{*}{ Orgánica } & \multirow{3}{*}{ Bandeja } & 5 & 4,4 & 4,3 & 3,6 & 3,1 & 5,0 & 5,0 & 5,0 & 5,0 & 5,0 & 4,5 & 3,0 & 3,0 \\
\hline & & 10 & 4,5 & 4,3 & 4,0 & 1,4 & 5,0 & 5,0 & 4,3 & 2,9 & 5,0 & 4,5 & 4,1 & 4,0 \\
\hline & & 20 & 1,6 & 2,1 & - & - & 5,0 & 4,6 & - & - & 5,0 & 3,3 & - & - \\
\hline & \multirow{3}{*}{ Bolsa } & 5 & 4,5 & 4,8 & 4,5 & 4,1 & 5,0 & 5,0 & 5,0 & 5,0 & 5,0 & 4,9 & 4,5 & 4,0 \\
\hline & & 10 & 4,4 & 4,9 & 4,3 & 3,5 & 5,0 & 5,0 & 5,0 & 4,3 & 5,0 & 5,0 & 4,5 & 4,1 \\
\hline & & 20 & 4,0 & 2,0 & - & - & 5,0 & 5,0 & - & - & 4,4 & 3,3 & - & - \\
\hline \multicolumn{15}{|l|}{ ANDEVA $^{1}$} \\
\hline F & & & $*$ & ns & ns & $*$ & ns & ns & ns & ns & ns & ns & $* * *$ & $* * *$ \\
\hline E & & & $* * *$ & $* * *$ & $* * *$ & $* * *$ & ns & $\mathrm{ns}$ & $* * *$ & $* * *$ & $* * *$ & $* * *$ & $* * *$ & $* * *$ \\
\hline $\mathrm{T}$ & & & $* * *$ & $* * *$ & ns & $* * *$ & ns & $* *$ & $* * *$ & $* * *$ & $* * *$ & $* * *$ & $* *$ & $\mathrm{~ns}$ \\
\hline $\mathrm{E}^{*} \mathrm{~T}$ & & & $* * *$ & ns & $\mathrm{ns}$ & $* * *$ & ns & $*$ & $* * *$ & $* * *$ & $* * *$ & ns & $*$ & $\mathrm{~ns}$ \\
\hline
\end{tabular}

${ }^{1}$ Valores cercanos a cinco semejan el producto recién cosechado.

Simbología: $\mathrm{ns}=(\mathrm{P}>0,05) ; *=(\mathrm{P}<0,05) ; * *=(\mathrm{P}<0,01) ; * * *=(\mathrm{P}<0,001) ;-=$ Lectura omitida por deterioro del producto.

en bandejas a $20{ }^{\circ} \mathrm{C}$ presentó algún daño microbiano. A partir de este periodo, el producto almacenado en bandejas a $10{ }^{\circ} \mathrm{C}$ presentó una acentuada pérdida de calidad, en relación con el daño microbiano y, después del décimo segundo día de almacenamiento, ya presentaba calificación inferior a cuatro para este aspecto. El palmito empacado en bolsas o bandejas, a temperatura de $5{ }^{\circ} \mathrm{C}$, no presentó daño microbiano importante hasta el final de su almacenamiento (15 días).

Los principales microorganismos observados en el palmito fueron los hongos Thielaviopsis basicola, $\mathrm{Pe}$ nicillium sp y Fusarium sp. El daño aparente causado por $T$. basicola se caracteriza por manchas deprimidas, ovaladas de aproximadamente 5 a $9 \mathrm{~mm}$ de largo y 2 a $5 \mathrm{~mm}$ de ancho, con el centro de coloración verdosa por el crecimiento del micelio y con apariencia acuosa alrededor (Figura 2a). La contaminación por Penicillium fue observada principalmente en las extremidades cortadas y se caracteriza por una mancha relativamente grande por el crecimiento del micelio del hongo, irregular y de color azul-verdoso (Figura 2). El daño causado por Fusarium es caracterizado por la presencia de manchas negras, aparentemente no deprimidas, con diámetro de aproximadamente 2 a $3 \mathrm{~mm}$ (Figura 2).

El palmito originado de parcelas en las cuales se aplicó el fertilizante orgánico presentó mayor emanación de olores extraños o desagradables $(\mathrm{P}<0,001)$, después de los 12 dea (Cuadro 3).

El empaque en bandejas y la temperatura superior a los $10{ }^{\circ} \mathrm{C}$, así como la combinación de ambos 


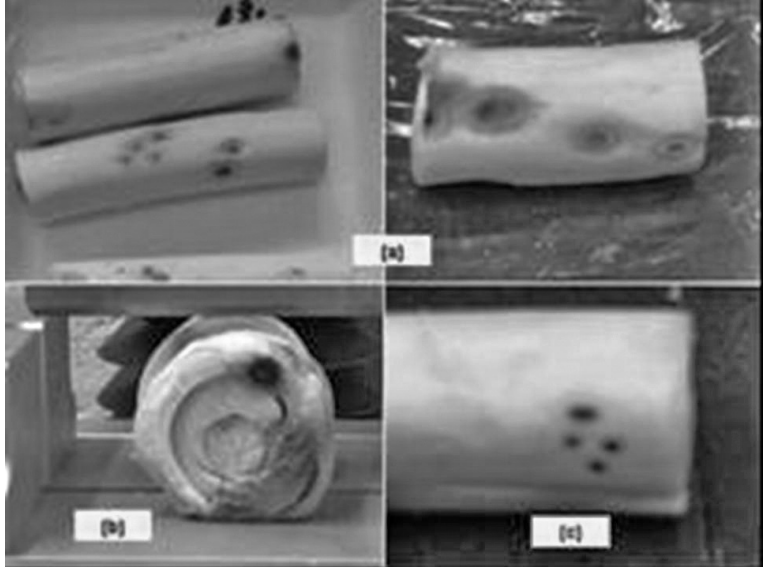

Figura 2. Hongos contaminantes del palmito fresco: (a) Thielaviopsis basicola, (b) Penicillium sp y (c) Fusarium sp. Costa Rica. 2005.

factores (interacción empaque $\mathrm{x}$ temperatura, $\mathrm{P}<$ $0,001)$ favorecieron la emanación de olores indeseables (Cuadro 3). La calidad del producto almacenado en bolsas a 5 y $10{ }^{\circ} \mathrm{C}$ fue superior en relación a la emanación de olores indeseables y no hubo riesgo de la calidad, de forma importante, hasta el final de su almacenamiento (15 días). Sin embargo, el producto almacenado en bandejas, aún a $5^{\circ} \mathrm{C}$, presentó malos olores a partir del día 12 en almacenamiento y a $10{ }^{\circ} \mathrm{C}$ tenía su calidad en riesgo en relación a la emanación de olores indeseables a partir del noveno día en almacenamiento. Los olores más fuertes detectados podrían calificarse como agrios o alcohólicos, producto de las transformaciones de carbohidratos.

\section{Textura}

La textura (dureza) promedio inicial, expresada en términos de resistencia al corte, fue de 41,0 $\pm 11,1$ $\mathrm{N}$ para el palmito fertilizado con abono orgánico $\mathrm{y}$ de 40,0 $\pm 12,3 \mathrm{~N}$ con abono químico. El tipo de fertilización no influyó sobre la textura del palmito. Sin embargo, el tipo de empaque, mostró su efecto en la textura, a partir del noveno día (Cuadro 4). La temperatura alta manifestó su efecto negativo a partir del sexto día, cuando se observó un incremento en la resistencia al corte (Figura 3). El efecto de la interacción empaque $\mathrm{x}$ temperatura también se hizo evidente $(\mathrm{P}<$ $0,001)$ y aquel producto almacenado en bandejas a 20 ${ }^{\circ} \mathrm{C}$ mostró su endurecimiento a partir del sexto día.
El producto empacado en bolsas y almacenado a $20{ }^{\circ} \mathrm{C}$ también presentó un serio deterioro en la textura (Cuadro 4). Sin embargo, el palmito almacenado a 5 ${ }^{\circ} \mathrm{C}$ en bolsas y bandejas no tuvo su calidad comprometida con relación a la textura del producto $\mathrm{y}$, además, el valor de fuerza de corte fue inferior al producto almacenado a $10{ }^{\circ} \mathrm{C}$ (Cuadro 4), lo que indica la importancia del almacenamiento del palmito al natural a baja temperatura.

\section{Color}

Como se mencionó en "Materiales y Métodos" el color fue evaluado considerando los parámetros "a", que representa las funciones rojo-verde $(+a,-a)$, "b", que representa las funciones amarillo-azul $(+b,-$ b) y "L", que representa las funciones blanco-negro o claro-oscuro (L) y que corresponden a las sensaciones que el cerebro humano recibe de los nervios ópticos.

Para el palmito recién cosechado, los valores pro-

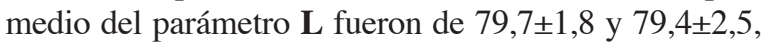
para el producto originado de cepas con fertilización química y orgánica, respectivamente, lo que indica un color más cercano al blanco. Sin embargo, el producto almacenado a $20{ }^{\circ} \mathrm{C}$ presentó importante pérdida de calidad con relación al color, ya que el palmito se oscureció a partir de los nueve día (Cuadro 5). Al final del período de almacenamiento (15 días), el producto almacenado a 5 y $10{ }^{\circ} \mathrm{C}$ y empacado en bolsa o bandeja no presentó importante variación en relación con la luminosidad.

En relación con parámetro "a", el palmito recién cosechado de las parcelas que recibieron fertilización química presentó un valor promedio de -0,36 $\pm 0,25$. Mientras que el palmito producido con fertilizante orgánico, presentó un valor promedio de -0,62 $\pm 0,08$; o sea, de una tonalidad más verdosa que el palmito producido con fertilizante inorgánico.

El palmito empacado en bandejas se presentaba más verdoso que el producto almacenado en bolsas de plástico (Cuadro 5). El efecto de la temperatura fue aún más marcado que el del empaque, en la inducción de cambios en el parámetro a, ya que a partir de los seis dea su efecto fue notorio $(\mathrm{P}<0,001)$ (Cuadro 5). También se logró determinar un efecto de la interacción empaque $\mathrm{x}$ temperatura $(\mathrm{P}<0,001)$ en este parámetro (Cuadro 5).

Se determinó, para el palmito recién cosechado de parcelas con fertilizante químico, un valor b promedio de $14,4 \pm 1,7$ y para aquel proveniente de parcelas con fertilizante orgánico, el valor promedio fue de 14,0 $\pm 1,8$. 
Cuadro 4. Textura (fuerza del corte) del palmito al natural, en función del tipo de fertilización, del empaque y de la temperatura de almacenamiento, a los seis, nueve, 12 y 15 días de almacenamiento. Costa Rica. 2005.

\begin{tabular}{|c|c|c|c|c|c|c|}
\hline \multirow{2}{*}{$\begin{array}{l}\text { Fertilización } \\
\text { (F) }\end{array}$} & \multirow{2}{*}{$\begin{array}{c}\text { Empaque } \\
\text { (E) }\end{array}$} & \multirow{2}{*}{$\begin{array}{c}\text { Temperatura } \\
{ }^{\circ} \mathbf{C}(\mathbf{T})\end{array}$} & \multicolumn{4}{|c|}{ Días en almacenamiento } \\
\hline & & & 6 & 9 & 12 & 15 \\
\hline \multirow[t]{6}{*}{ Química } & Bandeja & 5 & 36,4 & 49,9 & 46,4 & 43,2 \\
\hline & & 10 & 39,0 & 54,1 & 57,4 & 57,1 \\
\hline & & 20 & 68,7 & 97,1 & - & - \\
\hline & Bolsa & 5 & 43,8 & 39,7 & 40,3 & 37,0 \\
\hline & & 10 & 43,2 & 48,8 & 45,0 & 44,6 \\
\hline & & 20 & 52,5 & 46,7 & - & - \\
\hline \multirow[t]{6}{*}{ Orgánica } & Bandeja & 5 & 38,0 & 36,7 & 37,6 & 32,4 \\
\hline & & 10 & 38,6 & 48,5 & 50,2 & 46,1 \\
\hline & & 20 & 85,6 & 108,8 & - & - \\
\hline & Bolsa & 5 & 36,7 & 37,1 & 39,4 & 39,0 \\
\hline & & 10 & 44,1 & 44,3 & 41,3 & 47,0 \\
\hline & & 20 & 54,9 & 75,8 & - & - \\
\hline \multicolumn{7}{|l|}{ ANDEVA } \\
\hline F & & & ns & ns & ns & ns \\
\hline E & & & ns & $* * *$ & $* *$ & $* *$ \\
\hline $\mathrm{T}$ & & & $* * *$ & $* * *$ & $* * *$ & $* * *$ \\
\hline $\mathrm{E}^{*} \mathrm{~T}$ & & & $* * *$ & $* * *$ & $*$ & $\mathrm{~ns}$ \\
\hline
\end{tabular}

Simbología: $\mathrm{ns}=(\mathrm{P}>0,05) ; *=(\mathrm{P}<0,05) ; * *=(\mathrm{P}<0,01) ; * * *=(\mathrm{P}<0,001) ;-=$ Lectura omitida por deterioro del producto.

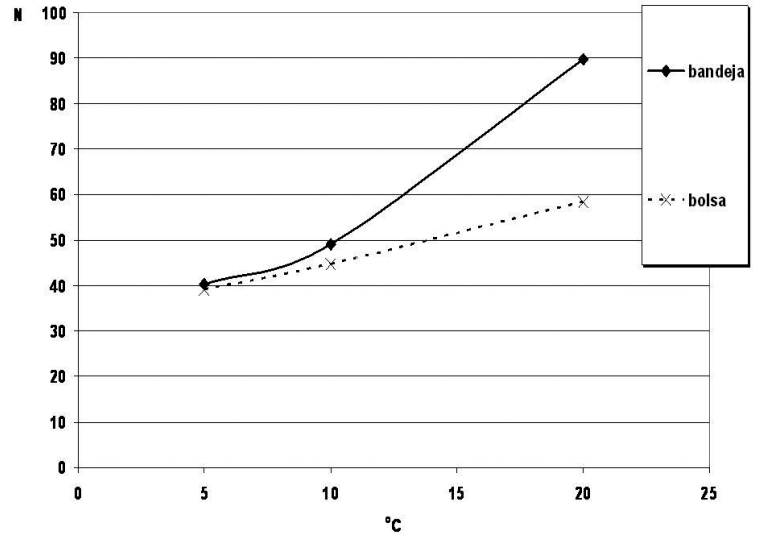

Figura 3. Textura promedio (resistencia al corte) del palmito en función de la temperatura de almacenamiento. Costa Rica. 2005.
La fertilización influyó en el parámetro b y al final del almacenamiento, el palmito de plantas fertilizadas con enmienda orgánica y almacenado en bandejas a $5{ }^{\circ} \mathrm{C}$ era más amarillento que aquel proveniente de plantas con fertilización inorgánica. De igual manera, el palmito empacado en bandejas se mostró más amarillento que el almacenado en bolsas de plástico (Cuadro 5). El almacenado a $20^{\circ} \mathrm{C}$ presentó pérdida de calidad por cambios en la pigmentación, ya que estaba más amarillento a los nueve días de almacenamiento, que el producto almacenado a temperaturas menores (Cuadro 5).

También se observó un efecto de la interacción empaque $\mathrm{x}$ temperatura $(\mathrm{P}<0,001)$ en el parámetro $\mathbf{b}$. Al final del período de almacenamiento (15 días), el producto almacenado en bandejas a 5 y $10^{\circ} \mathrm{C}$ se mostró más amarillento que aquel almacenado en bolsas y las mismas temperaturas. 
Cuadro 5. Parámetros de color del palmito al natural, en función del tipo fertilización, del empaque y de la temperatura a los seis, nueve, 12 y 15 días de almacenamiento. Costa Rica. 2005.

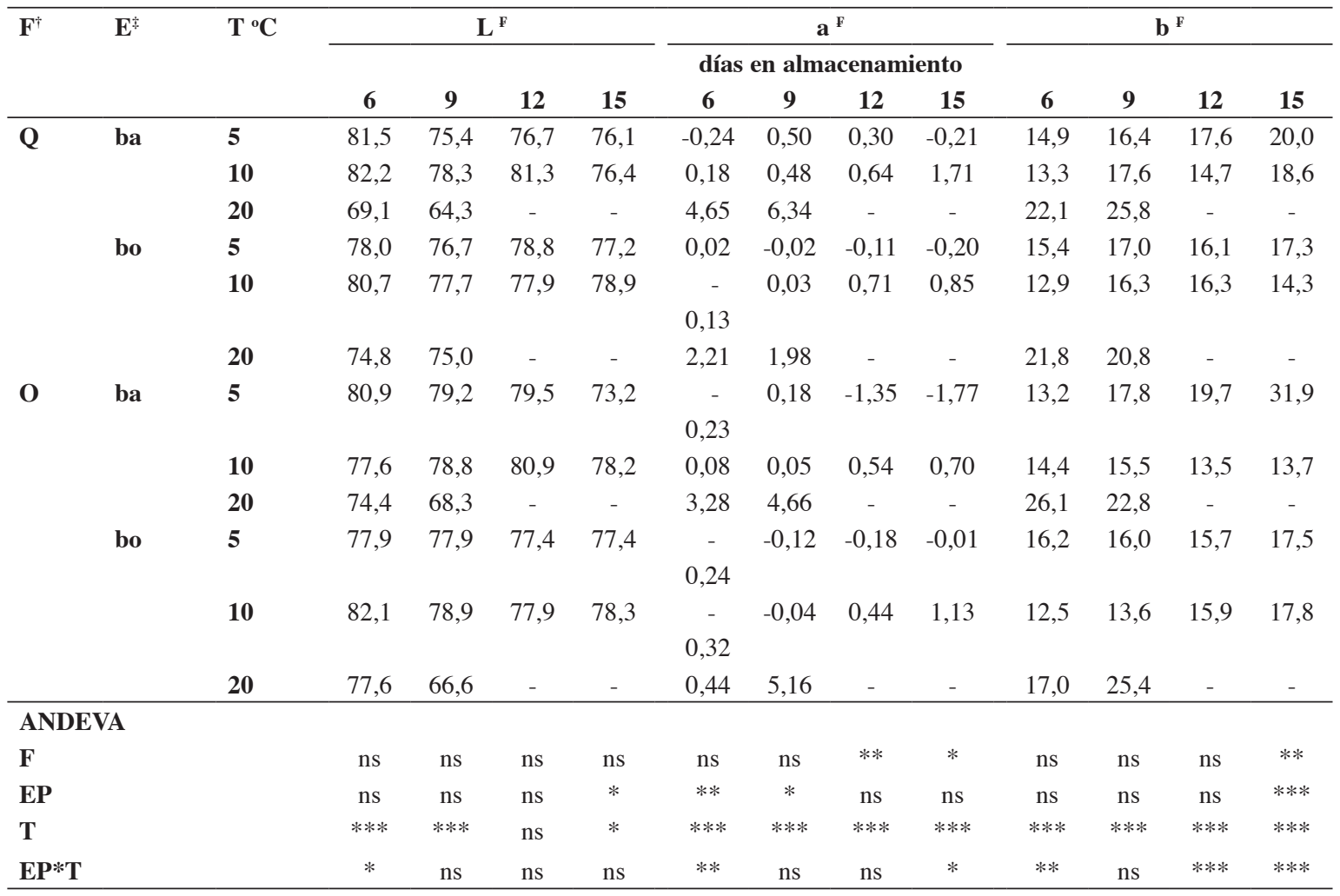

Simbología: 'Fertilización: $\mathrm{Q}=$ química, $\mathrm{O}=$ orgánica; ${ }^{\ddagger}$ Empaque: ba = bandeja, bo = bolsa plástica; $\mathrm{T}=$ temperatura en ${ }^{\circ} \mathrm{C}$; ${ }^{\mathrm{F}} \mathrm{L}=$ blanco $(100)-$ negro $(0) ; \mathrm{a} *=\operatorname{rojo}(+\mathrm{a})-\operatorname{verde}(-\mathrm{a}) ; \mathrm{b} *=\operatorname{amarillo}(+\mathrm{b})-\operatorname{azul}(-\mathrm{b}) ;{ }^{\mathrm{f}} \mathrm{ns}=(\mathrm{P}>0,05) ; *=(\mathrm{P}<0,05) ; * *=$ $(\mathrm{P}<0,01) ; * * *=(\mathrm{P}<0,001) ;-=$ Lectura omitida por deterioro del producto.

La diferenciación del color original en una muestra, estimada en este caso, mediante el parámetro $\Delta \mathrm{E}$ respondió a la aplicación de fertilizante, al final del período de almacenamiento (Cuadro 6). La fertilización orgánica propició la mayor alteración del color con respecto al original, y el producto mostró valores de color $(\Delta \mathrm{E})$ más altos que en el palmito fertilizado químicamente. El empaque y en mayor grado aún, la temperatura influyeron en los valores del color $(\Delta \mathrm{E})$, de manera que los valores más altos para esta variable correspondieron a aquellas muestras almacenadas en bandejas a $20{ }^{\circ} \mathrm{C}$ (Cuadro 6). El efecto de la interacción empaque $\mathrm{x}$ temperatura también se observaron y los mayores valores para este indicador de pérdida de calidad en las bandejas almacenadas a $20{ }^{\circ} \mathrm{C}$. Por otra parte, la estabilidad del color se mantuvo cuando el palmito se almacenó en bolsas plásticas a temperatura de 10 y $5{ }^{\circ} \mathrm{C}$ (Cuadro 6). El distanciamiento del color original de las muestras que se manifestó más pronunciadamente en las muestras almacenadas en bandejas, aún a temperaturas bajas, viene a ser básicamente un efecto debido al incremento en el parámetro b (Cuadro 5 ), que indica el amarillamiento del producto en esas condiciones.

\section{Acidez}

Inicialmente, el palmito fertilizado con enmienda orgánica presentó un $\mathrm{pH}$ inicial superior al observado en el cultivado con fertilizante químico $(6,45$ y 6,24, 
Cuadro 6. "Diferencia total de color" $(\Delta \mathrm{E})^{\dagger}$ del palmito al natural, en función del tipo fertilización, empaque y temperatura de almacenamiento, a los seis, nueve, 12 y 15 días de almacenamiento. Costa Rica. 2005.

\begin{tabular}{|c|c|c|c|c|c|c|c|c|}
\hline \multicolumn{2}{|c|}{ Fertilización - F } & \multicolumn{2}{|c|}{ Empaque - E } & \multirow{2}{*}{$\begin{array}{c}\text { Temperatura } \\
{ }^{\circ} \mathbf{C}-\mathbf{T}\end{array}$} & \multicolumn{4}{|c|}{ Días en almacenamiento } \\
\hline & & & & & 6 & 9 & 12 & 15 \\
\hline \multirow{6}{*}{ química } & \multirow{6}{*}{5,9} & & & 5 & 2,4 & 5,4 & 4,8 & 7,3 \\
\hline & & bandeja & 7,1 & 10 & 4,0 & 3,9 & 2,4 & 6,3 \\
\hline & & & & 20 & 14,3 & 21,0 & - & - \\
\hline & & & & 5 & 3,3 & 4,3 & 3,7 & 4,3 \\
\hline & & bolsa & 4,7 & 10 & 3,4 & 3,4 & 3,5 & 2,2 \\
\hline & & & & 20 & 9,6 & 8,7 & - & - \\
\hline \multirow{6}{*}{ orgánica } & \multirow{6}{*}{6,7} & & & 5 & 3,3 & 5,0 & 6,5 & 19,1 \\
\hline & & bandeja & 7,8 & 10 & 4,2 & 3,1 & 2,5 & 3,5 \\
\hline & & & & 20 & 14,5 & 15,9 & - & - \\
\hline & & & & 5 & 3,2 & 2,8 & 3,7 & 4,3 \\
\hline & & bolsa & 5,6 & 10 & 3,8 & 2,8 & 4,1 & 5,3 \\
\hline & & & & 20 & 4,7 & 18,5 & - & - \\
\hline \multicolumn{9}{|c|}{ ANDEVA } \\
\hline $\mathrm{F}$ & $\mathrm{ns}$ & & & & ns & ns & ns & $* *$ \\
\hline EP & $* * *$ & & & & $*$ & $\mathrm{~ns}$ & $\mathrm{~ns}$ & $* * *$ \\
\hline $\mathrm{T}$ & $* * *$ & & & & $* * *$ & $* * *$ & $* *$ & $* * *$ \\
\hline $\mathrm{EP} * \mathrm{~T}$ & $* * *$ & & & & $* *$ & ns & $* *$ & $* * *$ \\
\hline
\end{tabular}

${ }^{\dagger}$ con relación al color del palmito recién cosechado

* grado de significancia: ns, no significativo; * 5\%,** $1 \%$; *** $0,1 \%$

respectivamente). No obstante, a los 15 dea, el $\mathrm{pH}$ promedio del palmito con fertilizante orgánico fue más bajo que el del producto con fertilizante químico (Figura 4 y Cuadro 7).

El palmito almacenado a $5^{\circ} \mathrm{C}$ mantuvo un $\mathrm{pH}$ promedio mayor que aquel de las muestras almacenadas a temperaturas más altas. Las muestras almacenadas en bolsas, también mantuvieron el pH más alto que el de las muestras almacenadas en bandejas (Cuadro 7). Asimismo, la conservación del $\mathrm{pH}$ en función del tipo de empaque utilizado varió con la temperatura: el producto almacenado a $5{ }^{\circ} \mathrm{C}$ presentó mayor valor del $\mathrm{pH}$ cuando fue empacado en bandejas; mientras que el valor del $\mathrm{pH}$ del palmito almacenado a $10{ }^{\circ} \mathrm{C}$ fue mayor cuando se empacó en bolsas plásticas (interacción empaque $\mathrm{x}$ temperatura, $\mathrm{P}<0,001)$.

\section{DISCUSIÓN}

El consumo de alimentos naturales producidos sin agroquímicos convencionales o conservados sin

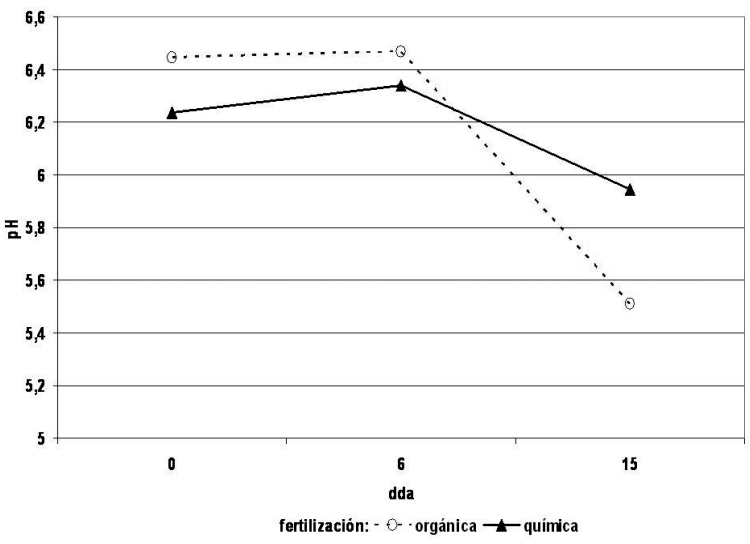

Figura 4. pH del palmito cultivado con fertilizante orgánico (O) y químico ( $\boldsymbol{\nabla})$ en diferentes días después de iniciado el almacenamiento. Costa Rica. 2005.

preservantes que amenazan la salud humana ha aumentado rápidamente en el mundo. Por otra parte, las organizaciones internacionales que promueven el 
Cuadro 7. $\mathrm{pH}$ del palmito al natural, en función del tipo de fertilización, empaque y temperatura de almacenamiento, a los 6 y 15 días en almacenamiento. Costa Rica. 2005.

\begin{tabular}{|c|c|c|c|c|c|}
\hline \multirow{2}{*}{$\begin{array}{l}\text { Fertiliza- } \\
\text { ción }(F)\end{array}$} & & \multirow{2}{*}{$\begin{array}{c}\text { Empaque } \\
\text { (E) }\end{array}$} & \multirow{2}{*}{$\begin{array}{c}\text { Temperatura } \\
{ }^{\circ} \mathbf{C}(\mathbf{T})\end{array}$} & \multicolumn{2}{|c|}{ pH } \\
\hline & & & & 6 & 15 \\
\hline \multirow{6}{*}{ química } & & & 5 & 6,38 & 6,39 \\
\hline & & bandeja & 10 & 6,20 & 5,18 \\
\hline & & & 20 & 6,21 & - \\
\hline & & & 5 & 6,37 & 6,29 \\
\hline & & bolsa & 10 & 6,37 & 5,93 \\
\hline & & & 20 & 6,50 & - \\
\hline \multirow{6}{*}{ orgánica } & & & 5 & 6,47 & 6,00 \\
\hline & & bandeja & 10 & 6,29 & 4,86 \\
\hline & & & 20 & 6,52 & - \\
\hline & & & 5 & 6,49 & 4,99 \\
\hline & & bolsa & 10 & 6,63 & 6,21 \\
\hline & & & 20 & 6,52 & - \\
\hline \multicolumn{6}{|l|}{ ANDEVA } \\
\hline F & ns & & & $* *$ & $* * *$ \\
\hline E & $* * *$ & & & $*$ & $* * *$ \\
\hline $\mathrm{T}$ & $* * *$ & & & ns & $* * *$ \\
\hline $\mathrm{E}^{*} \mathrm{~T}$ & $* * *$ & & & ns & $* * *$ \\
\hline
\end{tabular}

\$ grado de significancia: ns, no significativo; $* 5 \%$, ** $1 \%$; *** $0,1 \%$

desarrollo agropecuario de los países en vías de desarrollo, recomiendan la producción de alimentos orgánicos, como una oportunidad para incursionar competitivamente en el mercado internacional. Obviamente, las regulaciones de aquellos mercados selectivos y exigentes como los países miembros de la Unión Europea, para la importación de productos alimenticios son restrictivas (EUREPGAP 2005) y las instituciones nacionales deberán invertir en investigación que guíe a una producción orgánica controlada.

El presente trabajo se enmarca dentro de este contexto y representa un paso para producir el palmito de pejibaye en una forma amigable con el ambiente y con la salud humana. Los resultados obtenidos son halagadores en tanto que ponen en evidencia que el palmito para consumo al natural y producido orgánicamente puede ser almacenado hasta por unos 15 días, mediante el empaque del producto en bolsas plásticas de bajo costo y a una temperatura de $5{ }^{\circ} \mathrm{C}$. En lo que se refiere al tipo de empaque y temperatura de almacenamiento, el palmito de pejibaye no se comporta en forma muy diferente de otras hortalizas perecederas como la col (Sarantópoulos et al. 2003) y con mayor vida útil que otros productos como la lechuga almacenada a $2{ }^{\circ} \mathrm{C}$ (Freire Jr. et al. 2002) y el colinabo, almacenado a temperatura de $0{ }^{\circ} \mathrm{C}$ (Escalona et al. 2003). En aquellos casos como el brócoli (Cabezas y Richardson 1997), donde la temperatura de almacenamiento se ha reducido a $0{ }^{\circ} \mathrm{C}$, la vida útil se ha logrado incrementar hasta un máximo de cuatro semanas. En otros, como en el chile dulce, la vida en almacenamiento se ha mantenido hasta por tres semanas a $5{ }^{\circ} \mathrm{C}$ (González et al. 2004).

Evidentemente, la bandeja cubierta con la película de policloruro de vinilo fue inferior a la bolsa plástica para la preservación del palmito, aún a la temperatura de $5{ }^{\circ} \mathrm{C}$. La desventaja que se atribuye a la película de policloruro de vinilo fue el permitir el intercambio gaseoso que resultó dañino para la preservación del producto (Sigrist 2002, Rinaldi et al. 2005). La pérdida de peso fue una consecuencia evidente de la ineficacia de la bandeja para evitar la salida de vapor de agua, pues a la temperatura ambiente (aproximadamente $20{ }^{\circ} \mathrm{C}$ ) la pérdida de peso del producto almacenado en bandejas alcanzó casi el $4 \%$. Raíces de arracache (Arracacha xanthorriza Bancroft) almacenadas en bolsas plásticas y aproximadamente a $5{ }^{\circ} \mathrm{C}$, solo perdieron entre 0,05 y $0,46 \%$ de su peso, lo que hace suponer que la mayor permeabilidad de la película con que se cubran las bandejas sea el aspecto negativo. No obstante, otros ejemplos (Kehr 2002, Henz y Silva 1995), con el almacenamiento del chile dulce y la berenjena a bajas temperaturas no respaldan esta observación.

La pérdida de agua, además de causar una pérdida de peso induce a un incremento en la dureza y a cambios en el color natural (Garcia y Barrett 2002) y causó una reducción en la calidad del producto, principalmente cuando se almacena a temperatura alta.

La apariencia general del palmito y el daño microbiano se mantuvieron por encima del umbral (valor de cuatro), cuando el palmito se almacenó a $5^{\circ} \mathrm{C}$ y en bolsas plásticas, similar a los resultados de Clement et al. (1999) a una temperatura de $10{ }^{\circ} \mathrm{C}$. Que el daño 
microbiano no fuera un problema en aquel producto orgánico es estimulante para efectos de satisfacer las regulaciones internacionales que son estrictas en relación al daño microbiano (EUREPGAP 2005).

El valor de los olores extraños detectados en el palmito almacenado en las bolsas plásticas, incluyendo aquel producto de la fertilización orgánica, se mantuvieron en valores aceptables (superiores a cuatro), aún a los 15 días en almacenamiento. Los malos olores se asociaron al $\mathrm{pH}$ y a posibles diferencias cualitativas y/o cuantitativas en la composición química del producto. En lechuga, Souza et al. (2005) comprobaron una disminución en el contenido de azúcares solubles y un aumento en la concentración de proteína concomitantemente con el incremento en la cantidad de fertilizante orgánico aplicado. En melón también se han detectado diferencias en la concentración de azúcares en respuesta a diferentes combinaciones de fertilizantes químicos y orgánicos (Rodrigues et al. 2000).

En Costa Rica, se han encontrado diferencias en el $\mathrm{pH}$ del palmito, en respuesta a la variedad (De la Asunción 1992) y a la localidad, específicamente a la textura del suelo y a la fertilización (Pereira 2002). En Brasil, se han observado cambios en el pH del palmito al momento de la cosecha, en respuesta a la fertilización nitrogenada (Bovi et al. 2002) y a la localidad (Chaimsohn et al. 2002). Sin embargo, Oliveira et al. (2004) no encontraron diferencias en los valores de $\mathrm{pH}$ del palmito de plantas de pejibaye fertilizadas con boñiga, con y sin aplicación de abono químicos.

En general, el pH en todas las investigaciones anteriores varió de 5,51 a 6,78, ámbito dentro del cual se ubican los valores de $\mathrm{pH}$ detectados en palmito orgánico $(6,45)$ y en el palmito procedente de las parcelas con fertilización química $(6,24)$ recién cosechados. Este hecho conlleva a proponer una investigación enfocada a mantener la acidez inicial del palmito orgánico y evitar su rápida disminución que, a los 15 días en almacenamiento, mostró valores más bajos de $\mathrm{pH}$ que el palmito producido con fertilizante químico.

Los valores descriptivos del cambio de color del producto con respecto al original, en términos del valor $\mathbf{L}$, son similares a aquellos observados por Córdoba (1995) y Pereira (2002). El parámetro a tiende a ser muy variable a condiciones edáficas y ambientales (Córdoba 1995, Pereira 2002), sin embargo estos trabajos confirman nuestras observaciones de que el palmito proveniente de cepas que recibieron fertilizante orgánico son proclives a adquirir una tonalidad verdosa con el tiempo. El parámetro b, que es considerado de mayor importancia en términos de cambios de coloración del palmito en el tiempo (Pereira 2002) aunque variable con la humedad (Chaimsohn 2001) y con la fertilización y la textura del suelo (Pereira 2002), también confirman nuestros resultados que señalan una tendencia al amarillamiento del palmito con el tiempo, aún en condiciones apropiadas para la preservación del producto (bolsas plásticas y a $5^{\circ} \mathrm{C}$ ). Estos cambios en la tonalidad del producto aunque no tan graves para la comercialización de este producto propiamente acondicionado, en las primeras dos semanas de la cosecha, merecen también ser investigados para la obtención de un producto de mejor calidad.

\section{CONCLUSIONES Y RECOMENDACIONES}

Los resultados obtenidos en esta investigación preliminar sobre el acondicionamiento poscosecha del palmito de pejibaye, suministran información positiva para la eventual producción y preservación del producto para su comercialización como producto al natural y señala algunos aspectos que deben mejorarse para lograr este objetivo. El almacenamiento en bolsas a una temperatura de $5{ }^{\circ} \mathrm{C}$, permitió conservar el producto hasta por 15 días, con características todavía apropiadas para la comercialización. No obstante, es necesario investigar algunos aspectos que podrían llevar a la presentación de un producto orgánico con características sensoriales aún mejores que las obtenidas en este trabajo. El palmito podría poseer alguna ventaja comparativa para su preservación que lo hace hasta cierto punto comparable con la alcachofa (Cynara scolymus), en el sentido de que el producto útil está recubierto por varias capas protectoras. Se podría evaluar la posibilidad de almacenar el palmito con una o más capas foliares extra que podrían ayudar a preservar el producto en aspectos sensoriales y de apariencia. También es pertinente evaluar la conservación en bolsas plásticas a temperaturas menores a los $5{ }^{\circ} \mathrm{C}$. Es importante comparar la constitución química del palmito en respuesta a la fertilización y verificar a qué podrían obedecer los cambios de $\mathrm{pH}$ y la generación de olores indeseables en el palmito orgánico. La producción y comercialización del palmito orgánico 
fresco, como se hace con la lechuga y otras hortalizas que son más susceptibles a la pérdida de calidad en poscosecha, ofrece una oportunidad para incursionar competitivamente en el mercado internacional.

\section{AGRADECIMIENTOS}

Los autores agradecen al CNPq-Brasil, por el otorgamiento de la beca de doctorado al primer autor, sin la cual este trabajo no se hubiera realizado. Al Centro de Investigación en Granos y Semillas de la Universidad de Costa Rica (UCR) por la indispensable ayuda financiera y logística. Al Centro Nacional de Ciencia y Tecnología en Alimentos (CITA), a la Escuela de Tecnología de Alimentos de la Universidad de Costa Rica y a la Profesora Patricia Esquivel, por su inestimable apoyo en la realización de este trabajo. A la Vice Rectoría de Investigación de la UCR por el apoyo financiero. A Guillermo Solano por el imprescindible apoyo en el trabajo de campo.

\section{LITERATURA CITADA}

Anefalos, LC; Tucci, MLS; Modolo, VA. 2007. Uma visão sobre a pupunheira no contexto do mercado de palmito. Análises e indicadores do Agronegócio 2(7):1-6.

Bourne, MC. 2002. Food texture and viscosity: Concept and measurement. 2 ed. Academic Press, New York. 423 p.

Bovi, MLA; Tucci, MLS; Spiering, SH; Gozzi, SMGM; Oliveira, MAJ; Gallo, PB. 2002. Plant mineral fertilization affecting heart-of-palm processing. Acta Horticulturae 575(2): 643-650.

Cabezas, A; Richardson, DG. 1997. Modified atmosphere packaging of broccoli florets: effects of temperature and package types. Postharvest Horticulture Series - Department of Pomology, University of California (19):8 -15 (Abstract capturado en busqueda con EBSCO Host).

Calvo L, JM. 2000. Identificación de nichos de mercado para comercialización de palmito de pejibaye en el Gran Área Metropolitana de Costa Rica. Tesis de Lic. Administracion de Empresas Agropecuarias. Universidad Estatal a Distancia, San José, Costa Rica. 104 p.

Chaimsohn, FP. 2000. Cultivo de pupunha e produção do palmito. Viçosa, Editora Aprenda Fácil. 121 p.

Chaimsohn, FP. 2001. Cultivo de pupunha para palmito. Importância, mercado e aspectos biológicos e agronômicos. In: IAPAR. Curso sobre cultivo e processamento de palmito de pupunha. IAPAR, Londrina, p. 4-80. (IAPAR, Circular $\mathrm{n}^{\circ}$ 117).

Chaimsohn, FP; Morsbach, N; Durigan, ME; Treitny, MR; Gomes, EP. 2002. Desenvolvimento de pupunha (Bactris gasipaes Kunth) cultivada para palmito em diferentes regiões do Paraná. IAPAR/PRONAF, Londrina, 52 p. (IAPAR, Boletim Técnico ${ }^{\circ} 67$ ).

Chaimsohn, FP; Mora-Urpí, J; Villalobos-Rodríguez, E. 2007. Densidades de siembra, arreglos espaciales y fertilización en pejibaye (Bactris gasipaes cv 'Diamantes-10') para palmito. Agronomía Mesoamericana 18(2):205-220.

Clement, C; Manshardt, RM; Cavaletto, CG Defrank, J; Mood Jr., J; Nagai, NY; Fleming, K; Zee, F. 1996. Pejibaye heart-of-palm in Hawaii: From introduction to market. In: Janick, J. ed., Progress in new crops. ASHS Press, Arlington, VA. p. 500-507.

Clement, C; Bovi, MLA. 1999. Novos mercados para palmito - minimamente processado e 'pronto-para-uso' In: I Seminário do Agronegócio de Palmito de Pupunha na Amazônia Embrapa Rondônia, Sebrae-RO \& Cooperama, Porto Velho, RO, 11 A 13/08/1999.

Clement, CR; Santos, LA; Andrade, JS. 1999. Conservação de palmito de pupunha em atmosfera modificada. Acta Amazonica 29(3):437-445.

Córdoba S, I. 1995. Estudio sobre el efecto del empaque y la inmersión en la conservación de palmito fresco. Proyecto de graduación para optar al grado de Licenciado en Tecnología de Alimentos, Universidad de Costa Rica, San José, 94 p.

De la Asunción, R. 1992. Caracterización química del palmito de pejibaye (Bactris gasipaes Kunth), cultivares Utilis-Tucurrique y Putumayo-Yurimaguas. Proyecto de Graduación presentado para optar al grado de Licenciada en Tecnología de Alimentos, Universidad de Costa Rica, San José. 64 p.

De La Asunción, R; Flores, W; Mora-Urpí, J. 1995. Tecnologías apropiadas para el procesamiento de palmito de pejibaye. CITA-UCR, San José. p. 71-75.

Escalona, VH; Aguayo, E; Artes, F. 2003. Quality and physiological changes of fresh-cut kohlrabi. Acta Horticulturae 628(1):367-372.

EUREPGAP. 2005. Puntos de control y criterios decumplimiento frutas y hortalizas (en línea). Köln, EUREPGAP, 27 p. Consultado 28 abril 2005. Disponible: http://www. eurep.org/documents/webdocs/EUREPGAP_CPCC_ FP_V2-1_Oct04_SP_update_29Nov05.pdf

Ferreira, VLP. 1982. Caracterização físico-química e organoléptica de palmito proveniente da palmeira Guilielma 
gasipaes Bailey (pupunha) em relação ao palmito da palmeira Euterpe edulis Mart. (juçara). Tesis Mag. Sc. Escola Superior de Agricultura "Luiz de Queiroz", Piracibaba. 73 p.

Ferreira, VLP; Graner, M; Bovi, MLA; Draetta, IS; Paschoalino, JE; Shirose, I.. 1982. Comparação entre os palmitos Guilielma gasipaes Bailey (pupunha) e Euterpe edulis Mart. (juçara). I - Avaliações físicas, organolépticas e bioquímicas. Coleção ITAL 12:255-272.

Freire Júnior, M; Deliza, R; Chitarra, AB. 2002. Alterações sensoriais em alface hidropônica cv. Regina minimamente processada e armazenada sob refrigeração. Horticultura Brasileira 20(1):63-66.

Garrett, EH. 2002. Fresh-cut produce: tracks and trends. In: Lamikanra, O. Fresh-cut fruits and vegetables. Science, Technology an Market. CRC Press, Boca Raton. p. 1-10.

Garcia, E; Barrett, DM. 2002. Preservative treatments for fresh-cut fruits and vegetables. In: Lamikanra, O. ed. Fresh-cut fruits and vegetables. Science, Technology, and Market. CRC Press, Boca Raton. p. 267-303.

Good, H. s.f. Color measurement of cereal and cereal products. Consultado: 27 ene. 2005. Disponible en: http:// www.hunterlab.com/pdf/A5Cereal.pdf.

González A, GA; Ayala Zavala, JF, Ruiz-Cruz; S; AcedoFélix, E; Díaz Cinco, ME. 2004. Effect of temperature and modified atmosphere packaging on overall quality of fresh-cut bell peppers. Food Science \& Technology 37(8):817-827.

Henz, GP; Silva, C. 1995. Conservação de frutos de berinjela Cv. Ciça através de refrigeração e embalagem. Pesquisa Agropecuaria Brasileira 30(2):157-162.

Hunterlab. 1996. Hunter Lab Color Scale. Insight on Color 8(9):1-4.

Kader, AA. 2002. Quality parameters of fresh fruit and vegetable products. In: Lamikanra, O. ed. Freshcut fruits and vegetables. Science, Technology, and Market. CRC Press, Boca Raton. p. 11-20.

Kehr M, E. 2002. Susceptibilidad a daño por enfriamiento en poscosecha de pimiento y tratamientos para disminuir su efecto. Agricultura Técnica 62(4):509-518.

Kortbech-Olesen, R. 2002. The United States market for organic food and beverages. International Trade
Centre UNCTAD/WTO, 33 p. (en línea). Consultado: 26 dic. 2005. Disponible en: http://www.intracen.org/ mds/sectors/organic/foodbev.pdf

Mora-Urpí, J. 2002. Presente y futuro del palmito en Costa Rica. Agronomía Costarricense 26(2):95-100.

Oliveira, AP; Nascimento, JT; Souza, AP; Silva, IF; Alves, AU. 2004. Qualidade de palmito de pupunheira produzido com adubação organo-mineral, em Areia, PB. In: 44 Congresso Brasileiro de Olericultura, Campo Grande, 2004. CD Rom.

Pereira Q, JD. 2002. Perfil físico-químico del palmito de pejibaye (Bactris gasipaes Kunth) producido en Costa Rica. Proyecto de Graduación para optar al grado de Licenciado en Tecnología de Alimentos. San José, Universidad de Costa Rica. 68 p.

Rinaldi, MM; Benedetti, BC; Calore, L. 2005. Efeito da embalagem e temperatura de armazenamento em repolho minimamente processado. Ciência e Tecnologia de Alimentos 25(3):480-486.

Rodrigues, F; Oliveira, M; Pedrosa, JF; Bezerra Neto, F; Santos, MA; Negreiros, MZ. 2000. Rendimento e qualidade do melão Gold Mine adubado inorgânica e organicamente. Caatinga 13(1/2):59-65.

Santos, RHS; Silva, F; Casali, VWD; Conde, AR. 2001. Conservação pós-colheita de alface cultivada com composto orgânico. Pesquisa Agropecuária Brasileira, 36(3):521-525.

Sarantópoulos, CIGL; Oliveira, LM; Teles, CS; Coppelmans, SA. 2003. Efeitos da embalagem e da temperatura de estocagem na qualidade de couve minimamente processada. Brazilian Journal of Food Technology 6(2):185-190.

Sigrist, JMM. 2002. Estudos fisiológicos e tecnológicos de couve flor e rúcula minimamente processadas. Tesis Doctor, Escola Superior de Agricultura "Luiz de Queiroz", Piracicaba, Brasil. 112 p.

Souza, PA; Negreiros, MZ; Menezes, JB; Bezerra Neto, F; Souza, GLFM; Carneiro, CR; Queiroga, RCF. 2005. Características químicas de alface cultivada sob efeito residual da adubação com composto orgânico. Horticultura Brasileira 23(3):754-757.

Velázquez, C; Flores, W; Rivera, E. 2001. Ficha técnica para la producción de palmito fresco. San José, CITA, UCR. $14 \mathrm{p}$. 
